November 6, 2018

\title{
Semi-classical Laguerre polynomials and a third order discrete integrable equation
}

\author{
Paul E. Spicer \\ Katholieke Universiteit Leuven, Departement Wiskunde, Celestijnenlaan 200B B-3001 Leuven, \\ Belgium \\ Email: Paul.Spicer@wis.kuleuven.be \\ and \\ Frank W. Nijhoff \\ Department of Applied Mathematics, University of Leeds, Leeds LS2 9JT, U.K. \\ Email: frank.nijhoff@gmail.com
}

\begin{abstract}
A semi-discrete Lax pair formed from the differential system and recurrence relation for semiclassical orthogonal polynomials, leads to a discrete integrable equation for a specific semi-classical orthogonal polynomial weight. The main example we use is a semi-classical Laguerre weight to derive a third order difference equation with a corresponding Lax pair.
\end{abstract}

\section{Introduction}

The connection between semi-classical orthogonal polynomials and discrete integrable systems is well established. The earliest example of a discrete integrable system in semi-classical orthogonal polynomials can be attributed first to Shohat in 1939 [16, then second by Freud [10] in 1976. However it wasn't until the 1990's, when the focus within integrable systems shifted from continuous to discrete, that Fokas, Its, Kitaev, [6] gave this equation a name; discrete Painlevé I, (d-P $\mathrm{I})$.

Since then, other examples of discrete Painlevé equations have been found through exploring the recursive structures of different semi-classical orthogonal polynomial families, including semiclassical Hermite [15], semi-classical Laguerre 9] and semi-classical Charlier [17.

We define an orthogonal polynomial sequence $\left\{P_{n}(z)\right\}_{n=0}^{\infty}$ with respect to a weight function 
$w(z)$ on an interval $(a, b)$ as

$$
\int_{a}^{b} P_{n}(z) P_{m}(z) w(z) d z=h_{n} \delta_{n m}
$$

with the corresponding recurrence relation

$$
z P_{n}(z)=P_{n+1}+S_{n} P_{n}+R_{n} P_{n-1}
$$

for a monic orthogonal polynomial family $P_{n}(z)=z^{n}+p_{n n-1} z^{n-1}+p_{n n-2} z^{n-2}+\ldots$ From Bochner [2] we know that if $\left\{P_{n}(z)\right\}$ is a sequence of classical orthogonal polynomials, then $P_{n}(x)$ is a solution of the second-order differential equation

$$
\phi(z) \frac{d^{2} y}{d z^{2}}+\psi(z) \frac{d y}{d z}=\lambda_{n} y
$$

where $\phi(z)$ and $\psi(z)$ are fixed polynomials of degree $\leq 2$ and $\leq 1$ respectively, and $\lambda_{n}$ is a real number depending on the degree of the polynomial solution. As a consequence of this the weights of classical orthogonal polynomials satisfy a first order differential equation called the Pearson differential equation

$$
\frac{d}{d z}(\phi(z) w(z))=\psi(z) w(z),
$$

when the degrees of $\phi$ and $\psi$ satisfy $\operatorname{deg} \phi \leq 2$ and $\operatorname{deg} \psi=1$. However when the $\operatorname{deg} \phi>2$ and $\backslash$ or $\operatorname{deg} \psi>1$ then the weight function produces a class of semi-classical orthogonal polynomials.

Our approach to semi-classical orthogonal polynomials is to make use of the Laguerre method 11] (not to be confused with Laguerre orthogonal polynomials), which derives a pair of first order differential equations for a general class of orthogonal polynomials, after the reduction of continued fractions. The connection with semi-classical orthogonal polynomials occurs because we associate the system with a semi-classical weight function $w(x)$ of the polynomials with the Pearson equation (1.4). For convenience we choose to write the Pearson equation in the following form

$$
W(z) \partial_{z} w(z)=V(z) w(z)
$$

where $V(z)=\psi-\phi^{\prime}$ and $W(z)=\phi$. While our aim and approach is different, the Laguerre method has been used to find connections with integrable systems, including continuous Painlevé equations, recently. Magnus [14, found a continuous Painlevé equation of the sixth kind from the recurrence coefficients of a semi-classical Jacobi polynomial and Forrester and Witte [7, 8], found a Painlevé equation of the fifth kind, also using the Laguerre method, but one that has been extended to include bi-orthogonal polynomials.

Our work will consider a semi-classical Laguerre weight, similar to that used by [9]. The semiclassical Laguerre polynomials have not been as widely explored as the semi-classical Hermite 
polynomials, nor are they as complex as the semi-classical Jacobi polynomials. Thus, Laguerre polynomials are an appropriate choice for finding new discrete integrable systems.

In section 2 we use the so-called Laguerre method to derive the differential system for semiclassical (monic) orthogonal polynomials. We show how the compatibility between the differential system and the recurrence relation (1.2) leads to a semi-discrete Lax equation [13, from which discrete integrable systems can be derived for specific semi-classical orthogonal polynomial weights. In section 3 we choose the semi-classical Laguerre weight $l_{0}(x)=(x-t)^{\alpha} e^{-\left(a x+\frac{b}{2} x^{2}\right)}$ which leads to a coupled difference system and a corresponding third order nonlinear difference equation.

\section{The Laguerre Method}

We introduce a moment generating function, the Stieltjes function,

$$
f(z)=\int \frac{w(x)}{z-x} d(x)
$$

(Stieltjes transform of the orthogonality measure $w(x)$ ) then equations for $P_{n}$ can be summarized as

$$
f(z) P_{n}(z)=P_{n-1}^{(1)}(z)+\epsilon_{n}(z),
$$

where $P_{n-1}^{(1)}(z)$ is an associated polynomial to $P_{n}(z)$, with degree $n-1$. Although $\epsilon_{n}(z)$ is not a polynomial, we can define it as

$$
\epsilon_{n}(z)=\int \frac{P_{n}(x)}{z-x} w(x) d x .
$$

The polynomials $P_{n-1}^{(1)}(z)$, as well as the $\epsilon_{n}(z)$, satisfy the same recurrence relations (1.2), but with $P_{-1}^{(1)}(z)=0$. Additionally we have the following relations between $P_{n}, P_{n}^{(1)}$ and $\epsilon_{n}$

$$
\begin{aligned}
P_{n} P_{n-2}^{(1)}-P_{n-1} P_{n-1}^{(1)} & =-h_{n-1} \\
P_{n-1} \epsilon_{n}-P_{n} \epsilon_{n-1} & =-h_{n-1},
\end{aligned}
$$

which can be found using the Christoffel-Darboux identity. Since both $P_{n}(z)$ and $\epsilon_{n}(z)$ satisfy the recurrence relation (1.2) we can give an explicit form of $P_{n}(z)$ and $\epsilon_{n}(z)$ defined in terms of the recurrence relation's coefficients:

$$
\begin{aligned}
P_{n}(z) & =z^{n}-\left(\sum_{j=0}^{n-1} S_{j}\right) z^{n-1}+\sum_{j=1}^{n-1}\left(\sum_{k=0}^{j-1} S_{j} S_{k}-R_{j}\right) z^{n-2}+\cdots \\
\epsilon_{n}(z) & =h_{n}\left(\frac{1}{z^{n+1}}+\left(\sum_{j=0}^{n} S_{j}\right) \frac{1}{z^{n+2}}+\sum_{j=0}^{n}\left(R_{j+1}+\sum_{i=0}^{j} S_{j} S_{i}\right) \frac{1}{z^{n+3}}+\cdots\right) .
\end{aligned}
$$


Semi-classical orthogonal polynomials may be defined through a differential difference equation of the form

$$
W(z) \partial_{z} f(z)=V(z) f(z)+U(z)
$$

which comes from considering $W(z)\left(\partial_{z} f(z)\right)$ and the Pearson equation (1.5).

$$
\begin{aligned}
W(z)\left(\partial_{z} f(z)\right)=-\int \frac{W(z) w(x)}{(z-x)^{2}} d x & =-\int \frac{d}{d x}\left(\frac{1}{z-x} W(z) w(x)\right) d x+\int \frac{W(z)}{z-x} \partial_{x} w(x) \\
& =\int \frac{W(z)}{W(x)} V(x) \frac{1}{z-x} w(x) d x \\
& =V(z) f(z)+W(z) \int\left(\frac{V(x)}{W(x)}-\frac{V(z)}{W(z)}\right) \frac{w(x)}{z-x} d x
\end{aligned}
$$

On the first line we assume the first term reduces to zero because of parameter constraints and then we have that:

$$
U(z)=W(z) \int\left(\frac{V(x)}{W(x)}-\frac{V(z)}{W(z)}\right) \frac{w(x)}{z-x} d x,
$$

where $U(z)$ is a polynomial in $z$.

\subsection{The fundamental linear system for semi-classical orthogonal poly- nomials}

We start with the equation (2.2), differentiate it and multiply by $W$, so that we can then make use of the first order linear differential equation (2.6) (with the exception, that for this case we will consider the $x$ variable to be dominant).

$$
\begin{aligned}
W f \partial_{x} P_{n}+(V f+U) P_{n} & =W\left(\partial_{x} P_{n-1}^{(1)}+\partial_{x} \epsilon_{n}\right) \\
W \partial_{x} P_{n}\left(P_{n-1}^{(1)}+\epsilon_{n}\right)+V P_{n}\left(P_{n-1}^{(1)}+\epsilon_{n}\right)+U P_{n}^{2} & =W\left(\partial_{x} P_{n-1}^{(1)}+\partial_{x} \epsilon_{n}\right) P_{n}
\end{aligned}
$$

We then go about separating the polynomial expression $P_{n-1}^{(1)}$ and $\epsilon_{n}$ so we get the following two equivalent expressions, which we denote $\Theta_{n}$

$$
\begin{aligned}
\Theta_{n} & =W\left(\partial_{x} P_{n-1}^{(1)} P_{n}-\partial_{x} P_{n} P_{n-1}^{(1)}\right)-U P_{n}^{2}-V P_{n} P_{n-1}^{(1)}, \\
& =W\left(\partial_{x} P_{n} \epsilon_{n}-\partial_{x} \epsilon_{n} P_{n}\right)+V P_{n} \epsilon_{n},
\end{aligned}
$$

where $\Theta_{n}$ is a polynomial bounded by a constant. We try the same method again except this time we use $f P_{n-1}$, which is again differentiated and multiplied by $W$.

$$
\begin{aligned}
\partial_{x} f P_{n-1}+f \partial_{x} P_{n-1} & =\partial_{x} P_{n-2}^{(1)}+\partial_{x} \epsilon_{n-1} \\
V P_{n-1}\left(P_{n-1}^{(1)}+\epsilon_{n}\right)+U P_{n} P_{n-1}+W \partial_{x} P_{n-1}\left(P_{n-1}^{(1)}+\epsilon_{n}\right) & =W\left(\partial_{x} P_{n-2}^{(1)}+\partial_{x} \epsilon_{n-1}\right) P_{n}
\end{aligned}
$$


Again we separate the polynomial expression $P_{n-1}^{(1)}$ and $\epsilon_{n}$ to get a second object, which will be called $\Omega_{n}$ :

$$
\begin{aligned}
\Omega_{n} & =W\left(P_{n} \partial_{x} P_{n-2}^{(1)}-P_{n-1}^{(1)} \partial_{x} P_{n-1}\right)-V P_{n-1} P_{n-1}^{(1)}-U P_{n} P_{n-1} \\
& =W\left(\epsilon_{n} \partial_{x} P_{n-1}-P_{n} \partial_{x} \epsilon_{n-1}\right)+V \epsilon_{n} P_{n-1}
\end{aligned}
$$

We can express both $\Omega_{n}$ and $\Theta_{n}$ in terms of the recurrence coefficients by substituting the expressions for $P_{n}(2.5 \mathrm{a})$ and $\epsilon_{n}(2.5 \mathrm{~b})$ into $\Omega_{n}(2.11 \mathrm{~b})$ and $\Theta_{n}(2.8 \mathrm{~b})$.

$$
\begin{aligned}
\Theta_{n}= & W(x) h_{n}\left\{\left[\frac{1}{x^{n+1}}+\left(\sum_{j=0}^{n} S_{j}\right) \frac{1}{x^{n+2}}+\cdots\right] \times\left[n x^{n-1}-\left(\sum_{j=0}^{n-1} S_{j}\right)(n-1) x^{n-2}+\cdots\right]\right. \\
& \left.+\left[\frac{n+1}{x^{n+2}}+\left(\sum_{j=0}^{n} S_{j}\right) \frac{n+2}{x^{n+3}}+\cdots\right] \times\left[x^{n}-\left(\sum_{j=0}^{n-1} S_{j}\right) x^{n-1}+\cdots\right]\right\} \\
& +V(x) \times h_{n}\left[\frac{1}{x^{n+1}}+\left(\sum_{j=0}^{n} S_{j}\right) \frac{1}{x^{n+2}}+\cdots\right] \times\left[x^{n}-\left(\sum_{j=0}^{n-1} S_{j}\right) x^{n-1}+\cdots\right] \\
\Omega_{n}= & W(x)\left\{h_{n}\left[\frac{1}{x^{n+1}}+\left(\sum_{j=0}^{n} S_{j}\right) \frac{1}{x^{n+2}}+\sum_{j=0}^{n}\left(R_{j+1}+\sum_{k=0}^{j} S_{j} S_{k}\right) \frac{1}{x^{n+3}}+\cdots\right]\right. \\
& \times\left[(n-1) x^{n-2}-(n-2)\left(\sum_{j=0}^{n-2} S_{j}\right) x^{n-3}+(n-3) \sum_{j=1}^{n-2}\left(\sum_{k=0}^{j-1} S_{j} S_{k}-R_{j}\right) x^{n-4}+\cdots\right] \\
& +h_{n-1}\left[x^{n}-\left(\sum_{j=0}^{n-1} S_{j}\right) x^{n-1}+\sum_{j=1}^{n-1}\left(\sum_{k=0}^{j-1} S_{j} S_{k}-R_{j}\right) x^{n-2}+\cdots\right] \\
& \left.\times\left[\frac{n}{x^{n+1}}+\left(\sum_{j=0}^{n-1} S_{j}\right) \frac{(n+1)}{x^{n+2}}+\sum_{j=0}^{n-1}\left(R_{j+1}+\sum_{k=0}^{j} S_{j} S_{k}\right) \frac{(n+2)}{x^{n+3}}+\cdots\right]\right\} \\
& +V(x) \\
& \times h_{n}\left[\frac{1}{x^{n+1}}+\left(\sum_{j=0}^{n} S_{j}\right) \frac{1}{x^{n+2}}+\sum_{j=0}^{n}\left(R_{j+1}+\sum_{k=0}^{j} S_{j} S_{k}\right) \frac{1}{x^{n+3}}+\cdots\right] \\
& \times\left[x^{n-1}-\left(\sum_{j=0}^{n-2} S_{j}\right) x^{n-2}+\sum_{j=1}^{n-2}\left(\sum_{k=0}^{j-1} S_{j} S_{k}-R_{j}\right) x^{n-3}+\cdots\right] .
\end{aligned}
$$

These definitions will be particularly useful when we are looking at examples of specific semiclassical weights.

Since the recurrence relation (1.2) can be expressed in a matrix form

$$
\psi_{n+1}(x)=\left(\begin{array}{cc}
x-S_{n} & -R_{n} \\
1 & 0
\end{array}\right) \psi_{n}(x), \text { where } \psi_{n}(x)=\left(\begin{array}{c}
P_{n}(x) \\
P_{n-1}(x)
\end{array}\right)
$$

we collect the important relations we have derived so far and put them in a matrix form so that our intended differential system can be written as one expression. We begin with the two expressions 
(2.8a) and (2.11a), written in matrix form:

$$
\left(\begin{array}{cc}
P_{n-1} & -P_{n-2}^{(1)} \\
P_{n} & -P_{n-1}^{(1)}
\end{array}\right)\left(\begin{array}{c}
W \partial_{x} P_{n-1}^{(1)} \\
W \partial_{x} P_{n}
\end{array}\right)=\left(\begin{array}{c}
\Omega_{n}+V P_{n-1} P_{n-1}^{(1)}+U P_{n} P_{n-1} \\
\Theta_{n}+V P_{n} P_{n-1}^{(1)}+U P_{n}^{2}
\end{array}\right),
$$

which can easily be solved making use of (2.4a) to give:

$$
\left(\begin{array}{c}
W \partial_{x} P_{n-1}^{(1)} \\
W \partial_{x} P_{n}
\end{array}\right)=\frac{1}{h_{n-1}}\left(\begin{array}{cc}
P_{n-1}^{(1)} & -P_{n-2}^{(1)} \\
P_{n} & -P_{n-1}
\end{array}\right)\left(\begin{array}{c}
\Omega_{n}+V P_{n-1} P_{n-1}^{(1)}+U P_{n} P_{n-1} \\
\Theta_{n}+V P_{n} P_{n-1}^{(1)}+U P_{n}^{2}
\end{array}\right),
$$

so that we have two differential equations:

$$
\begin{aligned}
W \partial_{x} P_{n} & =\frac{1}{h_{n-1}}\left(\Omega_{n} P_{n}-\Theta_{n} P_{n-1}\right), \\
W \partial_{x} P_{n-1}^{(1)} & =\left(\Omega_{n} P_{n-1}^{(1)}-\Theta_{n} P_{n-2}^{(1)}+V h_{n-1} P_{n-1}^{(1)}+U h_{n-1} P_{n}\right) .
\end{aligned}
$$

Looking for a second differential relation for $P_{n}$, we take (2.15a) with a reduced index in conjunction with the recurrence relation (1.2), which leads to

$$
W\left(\partial_{x} P_{n-1}\right)=\frac{1}{h_{n-2}}\left(\Omega_{n-1} P_{n-1}-\frac{\Theta_{n-1}}{R_{n-1}}\left(\left(x-S_{n-1}\right) P_{n-1}-P_{n}\right)\right) .
$$

However we have no expression to remove the $x$ from the equation, so we consider the problematic part of the expression: $\left(x-S_{n}\right) \Theta_{n}=\left(x-S_{n}\right)\left(W\left(\epsilon_{n} \partial_{x}\left(P_{n}\right)-\partial_{x}\left(\epsilon_{n}\right) P_{n}\right)+V \epsilon_{n} P_{n}\right)$, which we expand using (1.2) and the differential of (2.4b) to get:

$$
\begin{aligned}
\left(x-S_{n}\right) \Theta_{n}= & W\left(-\partial_{x} \epsilon_{n}\left(P_{n+1}+R_{n} P_{n-1}\right)+\partial_{x} P_{n}\left(\epsilon_{n+1}+R_{n} \epsilon_{n-1}\right)\right) \\
& +V P_{n}\left(\epsilon_{n+1}+R_{n} \epsilon_{n-1}\right) \\
= & \Omega_{n+1}+R_{n} \Omega_{n}+V h_{n}
\end{aligned}
$$

This allows us to remove $x$ from (2.16) to give a second differential equation.

$$
W \partial_{x} P_{n-1}=\frac{1}{h_{n-1}}\left(\Theta_{n-1} P_{n}-\Omega_{n} P_{n-1}\right)-V P_{n-1}
$$

We now have a differential system

$$
W \partial_{x} \psi(x)=\frac{1}{h_{n-1}}\left(\begin{array}{cc}
\Omega_{n}(x) & -\Theta_{n}(x) \\
\Theta_{n-1}(x) & -\left(\Omega_{n}(x)+V(x) h_{n-1}\right)
\end{array}\right) \psi(x)
$$

where $\psi(x)=\left(\begin{array}{c}P_{n}(x) \\ P_{n-1}(x)\end{array}\right)$. Thus if we give the recurrence and differential equations in a semidiscrete Lax representation we have

$$
\begin{aligned}
\psi_{n+1}(x) & =L_{n}(x) \psi_{n}(x) \\
\partial_{x} \psi_{n}(x) & =M_{n}(x) \psi_{n}(x)
\end{aligned}
$$


where

$$
L_{n}=\left(\begin{array}{cc}
x-S_{n} & -R_{n} \\
1 & 0
\end{array}\right) \quad, \quad M_{n}=\frac{1}{W h_{n-1}}\left(\begin{array}{cc}
\Omega_{n}(x) & -\Theta_{n}(x) \\
\Theta_{n-1}(x) & -\left(\Omega_{n}(x)+V(x) h_{n-1}\right)
\end{array}\right) .
$$

Here we have identified the Lax matrices $L_{n}$ and $M_{n}$. So given a particular semi-classical weight we can identify the polynomials $V$ and $W$, which in turn lead to expressions for $\Theta$ and $\Omega$.

\subsection{Compatibility relations}

We now use the differential system (2.19) with the matrix form of the recurrence relation (2.13) in order to create a compatibility relation so that relations between $\Omega_{n}$ and $\Theta_{n}$ can be derived. Thus we consider the compatibility between the semi-discrete Lax pair, which leads to the semi-discrete Lax equation

$$
\partial_{x} L_{n}=M_{n+1} L_{n}-L_{n} M_{n}
$$

Equating this expression

$$
\begin{aligned}
\left(\begin{array}{ll}
1 & 0 \\
0 & 0
\end{array}\right)= & \frac{1}{W h_{n}}\left(\begin{array}{cc}
\Omega_{n+1}(x) & -\Theta_{n+1}(x) \\
\Theta_{n}(x) & -\left(\Omega_{n+1}(x)+V(x) h_{n}\right)
\end{array}\right)\left(\begin{array}{cc}
x-S_{n} & -R_{n} \\
1 & 0
\end{array}\right) \\
& -\frac{1}{W h_{n-1}}\left(\begin{array}{cc}
x-S_{n} & -R_{n} \\
1 & 0
\end{array}\right)\left(\begin{array}{cc}
\Omega_{n}(x) & -\Theta_{n}(x) \\
\Theta_{n-1}(x) & -\left(\Omega_{n}(x)+V(x) h_{n-1}\right)
\end{array}\right)
\end{aligned}
$$

we can identify two distinct relations

$$
\begin{aligned}
\left(x-S_{n}\right)\left(\frac{\Omega_{n+1}}{h_{n}}-\frac{\Omega_{n}}{h_{n-1}}\right) & =R_{n+1} \frac{\Theta_{n+1}}{h_{n+1}}-R_{n} \frac{\Theta_{n-1}}{h_{n-1}}+W \\
\left(x-S_{n}\right) \frac{\Theta_{n}}{h_{n}} & =\frac{\Omega_{n+1}}{h_{n}}+\frac{\Omega_{n}}{h_{n-1}}+V,
\end{aligned}
$$

which we can identify as being comparable with the Laguerre-Freud equations 1 .

Remark 2.1 We should point out that this system could be explored independent of orthogonal polynomials by simply setting $V=v_{0}+v_{1} x+v_{2} x^{2}+\ldots+v_{n} x^{n}$ and $W=w_{0}+w_{1} x+w_{2} x^{2}+\ldots+w_{n} x^{n}$ (where the $v_{j}, w_{j}$ are constants) and then see what difference equations are produced for different orders of $V$ and $W$. However since we are interested with the connections with semi-classical orthogonal polynomials, we will present a semi-classical weight and then determine $V$ and $W$. 


\section{A coupled difference equation and corresponding third order nonlinear equation}

This method can be demonstrated by using a semi-classical weight $l_{0}(x)=(x-t)^{\alpha} e^{-\left(a x+\frac{b}{2} x^{2}\right)}$ synonymous with the (associated) Laguerre orthogonal polynomials $l(x)=x^{\alpha} e^{-x}$. Our choice of deformations for this weight, involve altering the order of the polynomial in the exponential.

We first consider a deformation in the exponential part of the weight function, the semi-classical weight $w(x)=(x-t)^{\alpha} e^{-\left(a x+\frac{b}{2} x^{2}\right)}$ with $\alpha, a, b>0$ and where the support $S$ is an arc from $(t \rightarrow \infty)$. Then from the Pearson equation, we have

$$
V(x)=\alpha-(a+b x)(x-t) \quad, \quad W(x)=x-t .
$$

and from the consistency relations we have two non-trivial equations

$$
\begin{aligned}
& b\left(R_{n+1}+R_{n}\right)=-S_{n}\left[b S_{n}+(a-b t)\right]+(2 n+1+a t+\alpha), \\
& R_{n+1}\left[b\left(S_{n+1}+S_{n}\right)+(a-b t)\right]-R_{n}\left[b\left(S_{n}+S_{n-1}\right)+(a-b t)\right]=S_{n}-t .
\end{aligned}
$$

We consider this to be a nonlinear system in terms of the recurrence coefficients $R_{n}$ and $S_{n}$, which has the linear system (2.20) with the Lax pair:

$$
\begin{aligned}
L_{n} & =\left(\begin{array}{cc}
x-S_{n} & -R_{n} \\
1 & 0
\end{array}\right), \\
M_{n} & =\frac{1}{x-t}\left(\begin{array}{cc}
n-b R_{n} & \left(b x+a+b\left(S_{n}-t\right)\right) R_{n} \\
-\left(b x+a+b\left(S_{n-1}-t\right)\right) & b x^{2}+x(a-b t)+b R_{n}-n-\alpha-a t
\end{array}\right)
\end{aligned}
$$

for the associated semi-discrete Lax equation (2.21). This system can be called a discrete integrable system due to the existence of the corresponding linear problem, i.e., the Lax pair. In the strictest sense we cannot call the equation Painlevé since it is third order however there are other examples of third order nonlinear difference equations that are known to be integrable [4] .

Writing this system in matrix form

$$
\begin{aligned}
& \left(\begin{array}{cc}
b & b \\
b\left(S_{n+1}+S_{n}\right)+(a-b t) & -\left[b\left(S_{n}+S_{n-1}\right)+(a-b t)\right]
\end{array}\right)\left(\begin{array}{c}
R_{n+1} \\
R_{n}
\end{array}\right) \\
= & \left(\begin{array}{c}
-S_{n}\left(b S_{n}+(a-b t)\right)+(2 n+1+a t+\alpha) \\
S_{n}-t
\end{array}\right)
\end{aligned}
$$


allows us to solve the system in terms of $R_{n+1}$ and $R_{n}$ and hence we can find a third order difference equation in $S_{n}$

$$
\begin{aligned}
& \left\{\left(S_{n}+S_{n-1}+\left(\frac{a}{b}-t\right)\right)\left(S_{n}\left(S_{n}+\left(\frac{a}{b}-t\right)\right)\right)-\frac{1}{b}(2 n+1+a t+\alpha)\left(\left(S_{n}+S_{n-1}\right)\right.\right. \\
+ & \left.\left.\left(\frac{a}{b}-t\right)\right)-\left(S_{n}-t\right)\right\} \times\left\{-2\left(S_{n+1}+\frac{a}{b}-t\right)-\left(S_{n}+2 S_{n+1}+S_{n+2}\right)\right\} \\
= & \left\{\left(S_{n+2}+S_{n+1}+\left(\frac{a}{b}-t\right)\right)\left(S_{n+1}\left(S_{n+1}+\left(\frac{a}{b}-t\right)\right)\right)-\frac{1}{b}(2 n+1+a t+\alpha)\left(\left(S_{n+2}+S_{n+1}\right)\right.\right. \\
& \left.\left.+\left(\frac{a}{b}-t\right)\right)-\left(S_{n+1}-t\right)\right\} \times\left\{-2\left(S_{n}+\frac{a}{b}-t\right)-\left(S_{n-1}+2 S_{n}+S_{n+1}\right)\right\} .
\end{aligned}
$$

Alternatively by letting $S_{n}=Q_{n}-Q_{n-1}$ in (3.2), we are led to an alternative third order difference equation in $Q_{n}$ that includes an extra parameter $c$

$$
\begin{aligned}
& b\left(\frac{Q_{n}-(n+1) t+c}{a-b t+b\left(Q_{n+1}-Q_{n-1}\right)}+\frac{Q_{n-1}-n t+c}{a-b t+b\left(Q_{n}-Q_{n-2}\right)}\right) \\
=- & -\left(Q_{n}-Q_{n-1}\right)\left[b\left(Q_{n}-Q_{n-1}\right)+a-b t\right]+(2 n+1+a t+\alpha),
\end{aligned}
$$

(where $c$ is the constant of integration).

\section{Conclusion and Outlook}

Given a class of semi-classical orthogonal polynomials (Hermite, Laguerre and Jacobi), we can identify a semi-discrete Lax pair and thus an associated discrete integrable system. Using the Laguerre weight $l_{0}(x)=(x-t)^{\alpha} e^{-\left(a x+\frac{b}{2} x^{2}\right)}$, we found a new coupled discrete integrable system, which is first order in $R$ and second order in $S$. Combining the two equations gives a third order difference equation in $S$ or a third order difference equation in the new variable $Q$. Since we were only interested in the connections with discrete Painlevé equations, we have omitted to look at the additional $t$-differential equation (which appears as a consequence of the $t$ parameter in the weight function). It is likely that we could use the $t$-differential equation to find a continuous Painlevé equation related to our semi-classical Laguerre weight.

While we have chosen to look at a simple deformation of the classical orthogonal polynomial weight associated Laguerre, this scheme can of course be used for deriving a multitude of discrete integrable systems, through choosing an appropriate classical weight function. While we have made some progress in this regard, finding the corresponding continuous equation is not always possible. Working with further examples has shown that when looking for continuum limits, the choice of a semi-classical weight function must be of a particular form.

In this paper we have applied the Laguerre method to a family of classical orthogonal polynomials, but we expect it is possible that the Laguerre method can be used with other classes 
of orthogonal polynomials, such as the discrete, multiple or q-orthogonal polynomials. We would need to alter the method appropriately, such as choosing an analogue for the Pearson equation (since we are using the "classical Pearson equation" for classical orthogonal polynomials). Thus, we could derive a similar scheme for q-orthogonal polynomials given the q-Pearson equation, where a natural extension of this would be to consider the q-Laguerre orthogonal polynomials.

\section{Acknowledgements}

Paul Spicer wishes to thank Nalini Joshi for numerous discussions on integrable lattice equations, and for advice and encouragement. He wishes to thank Pavlos Kassotakis for proof reading the paper and useful insights. The author is supported by the Australian Research Council Discovery Project Grant \#DP0664624.

\section{References}

[1] S. Belmehdi and A. Ronveaux Laguerre-Freuds equations for the recurrence coefficients of semi-classical orthogonal polynomials, J. Approx. Theory 76 (1994) 351-368.

[2] S. Bochner, Über Sturm-Liouvillesche Polynomsysteme, Math. Zeit. 29 (1929) 730-736.

[3] E.B. Christoffel, Über die Gaussische Quadratur und eine Verallgemeinerung derselben, Journal für die reine und angewandte Mathematik, vol. 55 (1858), pp 61-82.

[4] B. Grammaticos, F. W. Nijhoff and A. Ramani, Chapter 7 Discrete Painlevé Equations in: R. Conte (Ed.), The Painlevé Property, Springer-Verlag, New York, 1999.

[5] G. Darboux, Mémoire sur l'approximation des fonctions de trés grands nombres, Journal de Mathématiques, (3), vol.4 (1878), pp. 5-56,377-416.

[6] A.S. Fokas, A.R. Its and A.V. Kitaev, Discrete Painlevé Equations and their Appearance in Quantum Gravity, Comm. Math. Phys. 142 (1991) 313-344.

[7] P.J. Forrester and N.S. Witte, Bi-orthogonal polynomials on the Unit Circle, Regular SemiClassical Weights and Integrable Systems, math.CA/0412394.

[8] P.J. Forrester and N.S. Witte, Discrete Painlevé Equations for a class of $P_{V I} \tau$-functions given as $U(N)$ averages, math.PH/0412065. 
[9] P.J. Forrester and N.S. Witte, The distribution of the first eigenvalue spacing at the hard edge of the Laguerre unitary ensemble, Kyushu Journal of Mathematics, 61, 457-526, 2007.

[10] G. Freud, On the coefficients in the recursion formulae of Orthogonal Polynomials, Proc. Roy. Irish Acad. Sect. A 76 (1976) 1-6.

[11] E. Laguerre Sur la réduction en fractions continues d'une fonction qui satisfait à une équation linéaire du premier ordre à coefficients rationnels, Bulletin de la S.M.F., tome 8 (1880), p.2127. http://www.numdam.org/item?id=BSMF 18808210

[12] E. Laguerre, Sur la réduction en fractions continues d'une fraction qui satisfait à une équation différentielle linéaire du premier ordre dont les coefficients sont rationnels J. Math. Pures Appl. (4) 1 (1885) 135-165 = pp. 685-711 in Oeuvres Vol. II, Chelsea, New York 1972.

[13] P.D. Lax, Integrals of nonlinear equations of evolution and solitary waves, Commun. Math. Phys. 21 (1968) 467-490.

[14] A.P. Magnus, Painlevé-type differential equations for the recurrence coefficients of semiclassical polynomials, J. Comput. Appl. Math. 57 (1995) 215-237. (math.CA/9307218)

[15] A.P. Magnus, Freud equations for orthogonal polynomials as discrete Painlevé equations in: Peter A. Clarkson, Frank W. Nijhoff (Eds.), Symmetries and Integrability of Difference Equations, Cambridge University Press, London Mathematical Society Lecture Note Series, vol. 255, 1999, pp. 228-243.

[16] J. A. Shohat, A Differential equation for Orthogonal Polynomials, Duke Math. J. 51939 401-417.

[17] W. Van Assche and M. Foupouagnigni, Analysis of non-linear recurrence relations for the recurrence coefficients of generalized Charlier polynomials, J. Nonlinear Math. Phys. 10 (2003), suppl. 2, 231237. 